\title{
Generation and enhancement of SRS sub-nanosecond pulses in self-focusing organic substances
}

\author{
O.Iu. Isaenko ${ }^{1}$, A.I. Ivanisik ${ }^{1}$, P.A. Korotkov ${ }^{1}$ and G.V. Ponezha ${ }^{2}$ \\ ${ }^{1}$ Taras Shevchenko Kyiv National University, \\ 4g, Academician Glushkov Avenue, 03127 Kyiv, Ukraine \\ Phone: +38 (044) 526-0570; e-mail: aivan@univ.kiev.ua \\ ${ }^{2}$ National Academy of Statistics, Accounting and Auditing, \\ 1, Pidgirna str., 04107 Kyiv, Ukraine \\ Phone: +38(044) 526-2778; e-mail:ponezha@mail.ru
}

\begin{abstract}
The possibility to use self-focusing organic substances in order to create highly efficient transformers of laser radiation based on stimulated Raman scattering has been researched. The proposed and implemented optical schemes are suitable for generation of seed sub-nanosecond Stokes pulses, due to dynamics of self-focusing, and the compression of giant pulses of multimode laser with high $(>20)$ increase in the intensity at a shifted frequency.
\end{abstract}

Keywords: laser, stimulated Raman scattering, self-focusing.

Manuscript received 31.01.14; revised version received 02.04.14; accepted for publication 12.06.14; published online 30.06.14.

\section{Introduction}

SRS (stimulated Raman scattering) can be successfully used in transformers of laser radiation for frequency tuning, pulse compression and improvement of optical beam quality $[1,2,3]$. However, SRS-active materials that have practical use are limited to $\mathrm{H}_{2}$ and $\mathrm{CH}_{4}$ gases (at pressure of $\sim 10 \mathrm{~atm}$ ) or crystals $\mathrm{Ba}\left(\mathrm{NO}_{3}\right)_{2}$, $\mathrm{KGd}\left(\mathrm{WO}_{4}\right)_{2}$. Such available, optically stable and easy to use materials as self-focusing liquids (benzene, toluene, etc.), in which historically first SRS was observed [4], were declined because of unsuccessful attempts to create the highly efficient quantum electronics devices on their basis. This narrows the range of radiation frequencies that can be obtained from a laser, and thus reduces the value of SRS as a method of frequency tuning.

An experimental threshold of SRS in self-focusing liquids is defined by the critical power of self-focusing [5] and is achieved quite easily. However, there are significant complications in achieving the high-energy conversion efficiency of laser radiation. Generally, the conversion efficiency in self-focusing liquids at the first Stokes SRS component does not exceed approximately $20 \%$ under the excitation by a giant pulse of a multimode laser. For all Stokes components, the conversion efficiency is close to $30 \%$. This situation has been classified as an effect of "absolute saturation of SRS" [6]. However, the nature of the effect remains unclear. The main problem is the effect of the smallscale self-focusing.

In our opinion, SRS-based transformers with a high conversion efficiency can be still created in self-focusing liquids, but in two-stage schemes. Namely, an SRS seed pulse is generated in the first cell above the threshold of self-focusing, and then it is enhanced below the threshold of self-focusing in the second cell. Beside achieving the high overall conversion efficiency in the two-cell scheme, it is possible to reduce a pulse duration in the first cell, using dynamics of self-focusing process.

It should be noted that the results of solving the problem of increasing the SRS effectiveness in selffocusing liquids are closely linked to the availability of 
exciting lasers. In practice, it is important to solve a complex problem: the efficient conversion of the available multimode laser radiation and, at the same time, improvement of the radiation optical quality. The above circumstances stimulated the experimental research described in this work.

Thus, the work is aimed to obtain the seed subnanosecond pulses in self-focusing SRS-active liquids under the excitation by multimode Q-switched laser and to use them in the gain stage with a high conversion efficiency.

\section{Experimental}

The multimode ruby laser $(1.9 \%$ concentration $\mathrm{Cr}$ in $\alpha$ $\mathrm{Al}_{2} \mathrm{O}_{3}$ crystal, diameter/length of crystal $0.8 \mathrm{~cm} / 12 \mathrm{~cm}$ ) with the resonator of $35-\mathrm{cm}$ length and passive Q-switching (gallium-phthalocyanine chloride in chlorobenzene) was used. The resonator consisted of a $100 \%$ mirror and the crystal exit plane with the reflectivity of $7.7 \%$. The resonator has provided generation of short and powerful pulses [7].

The laser radiated pulses with the wavelength $0.6943 \mu \mathrm{m}$, energy $0.6 \mathrm{~J}$, and duration $\tau_{L}=30 \mathrm{~ns}$ at the half level. The root mean square of the pulse energy variation was $5 \%$. An envelope of the laser pulses, recorded by $\mathrm{C} 7-10 \mathrm{~B}$ oscillograph, was smooth.

The pulse spectrum was measured by a Fabry-Pérot interferometer with a ring thickness of $30 \mathrm{~mm}$ and sharpness 20. The half-width of the pulse spectrum was $0.01 \mathrm{~cm}^{-1}$, which is less than the spectral distance $0.014 \mathrm{~cm}^{-1}$ between two longitudinal modes of the empty laser cavity. The laser beam at an intersection was heterogeneous with numerous $\left(\sim 10^{2}\right)$ maxima and minima in the intensity.

Without addition focusing, this laser provided the excess of the SRS threshold by 3.5 times in the toluenefilled cell of $25-\mathrm{cm}$ length. The conversion efficiency into all the Stokes components, propagating in the direction of the excitation beam, was approximately $20 \%$. Forward SRS-pulse duration ranged from 15 up to $25 \mathrm{~ns}$, which is no less than the half duration of the laser pulse.

To obtain and register the Stokes SRS pulses from a single focal region of self-focusing, an optical scheme shown in Fig. 1 was used. Laser radiation in the optical scheme (Fig. 1) partially $(0.8 \%)$ passed through the diaphragm and reached the $25-\mathrm{cm}$ toluene-filled cell. Optical filter $\mathrm{C} 3 \mathrm{C}-20$ after the cell absorbed the laser radiation. The scattered light after passing through the filter (mainly the first Stokes SRS) entered the registration scheme. The registration scheme consisted of a photovoltaic vacuum cell ФК-19.000 with the rise time (from 0.1 to 0.9 of the maximum value of the current pulse) less than $0.7 \mathrm{~ns}$, an oscillograph $\mathrm{C} 7-10 \mathrm{~B}$ with a signal delay line and energy meter ИМО-2H. Glass plates with the reflectivity $12 \%$ at $45^{\circ}$ created signals for the energy meter.

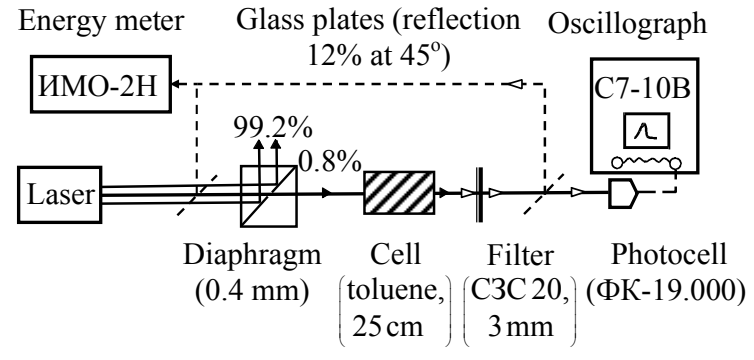

Fig. 1. Optical scheme for generation of seed sub-nanosecond pulse and for investigation of SRS effectiveness in a single focal region of self-focusing.

The diaphragm (Fig. 1) was formed by two $90^{\circ}$ prisms, which hypotenuse surfaces had an optical contact of limited area with glycerin. The surface of the optical contact had a round shape and the diameter $D \approx 0.4 \mathrm{~mm}$. This diaphragm has an advantage compared to the conventional one. Namely, at the powerful light pulse ( $20 \mathrm{MW}$ from the used laser), the optical breakdown was prevented on its boundary. In addition, the laser radiation, which does not pass the diaphragm $(99.2 \%)$ under the total internal reflection, can be used in the gain channel.

The diameter $D=0.4 \mathrm{~mm}$ of the diaphragm was chosen being based on the fact that the laser beam in toluene tends to fragment into separate filaments that self-focus independently. Applying the registration scheme to obtain the spatial-angular spectra and the calculation procedure described in the works $[8,9]$, it was determined that the average diameter of these filaments at the cell entrance was $d \approx 0.23 \mathrm{~mm}$ without the diaphragm. With the diameters ratio of $D / d<2$, there is a high probability that the only separate self-focusing focal point is formed simultaneously, and, thus, formation of many independent sources of SRS with different phases was prevented.

The 5-cm distance between the diaphragm and cell was selected experimentally to make sure that the SRS threshold is exceeded for each laser pulse, under the given non-stability of spatial and temporal characteristics of the used multimode laser.

When the distance between the diaphragm and cell has been increased up to $10 \mathrm{~cm}$, the threshold of SRS was not achieved. This is the consequence of the beam diffraction on the path between the diaphragm and cell. As a result of diffraction, an area of self-focusing was not formed.

Laser radiation passed through the diaphragm with the energy $4 \mathrm{~mJ}$. Under these conditions, the pulse energy of SRS was $0.15 \mathrm{~mJ}$.

Fig. 2 shows oscillograms of the laser (a) and the first Stokes SRS (b) pulses without the diaphragm, and of the laser (c) and the SRS (d) pulses after the diaphragm. To emphasize the differences in the shape and duration, all the pulses in Fig. 2 were normalized in 
such a manner that their amplitudes were the same. The oscillograms of laser pulses after the diaphragm (Fig. 2c) have almost $50 \%$ modulation with the period of about $7 \mathrm{~ns}$. Note that, for the laser resonator length of $35 \mathrm{~cm}$, the beating of longitudinal modes would lead to a modulation with a period of $2.3 \mathrm{~ns}$. So, this modulation should be attributed to the beating of transverse modes or deformation of the mode structure of the beam in the generation process.

The oscillogram of SRS pulse after the diaphragm (Fig. 2d) shows that a sub-nanosecond pulse with the duration close to $0.5 \mathrm{~ns}$ at the half level of intensity can be obtained. Quite often, one or two additional pulses were observed after the first pulse of SRS. They were usually longer and had a low amplitude. The period of these pulses closely correlates with that of the oscillation in the laser radiation intensity after the diaphragm (Fig. 2c). To enhance the obtained SRS pulses (Fig. 2d), the optical scheme shown in Fig. 3 was used. The seed SRS pulse twice passed a toluene-filled 1-m cell towards the main portion $(99.2 \%)$ of the laser beam. Optical filter ПC7 absorbed SRS radiation to prevent a feedback effect.
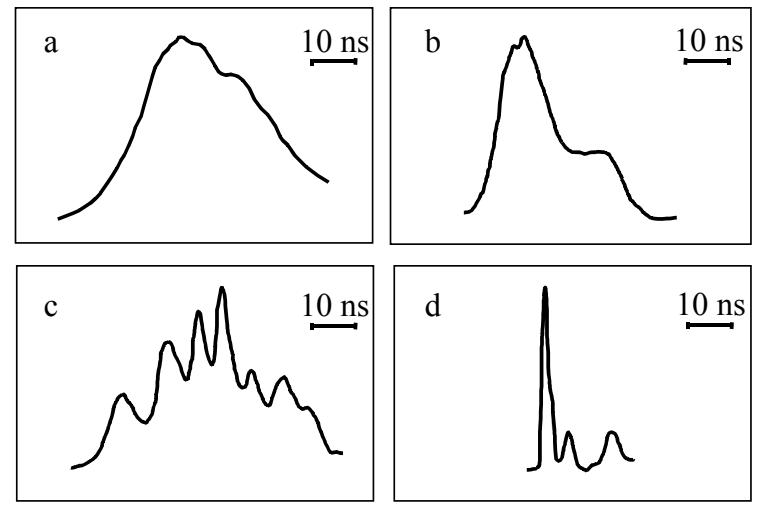

Fig. 2. The oscillograms of pulses: laser $(a)$ and SRS $(b)$ pulses without the diaphragm; laser $(c)$ and SRS $(d)$ pulses after the diaphragm.

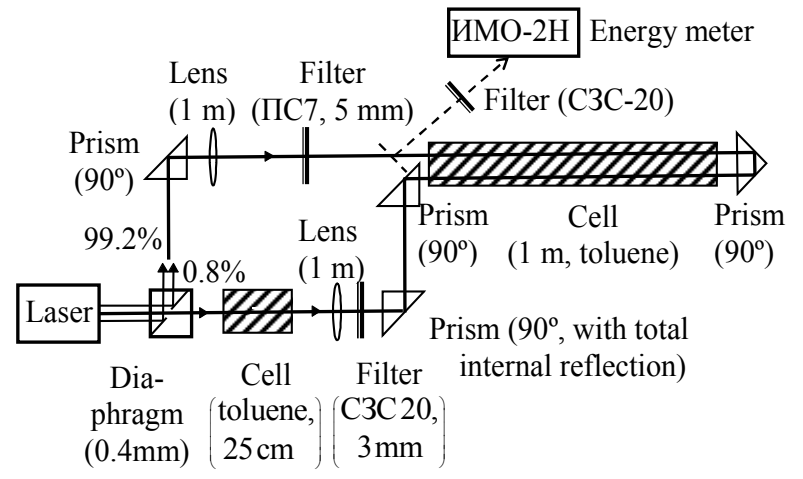

Fig. 3. Optical scheme for enhancement of SRS pulse from a single focal region of self-focusing in toluene (focal distance of lenses is $1 \mathrm{~m}$ ).

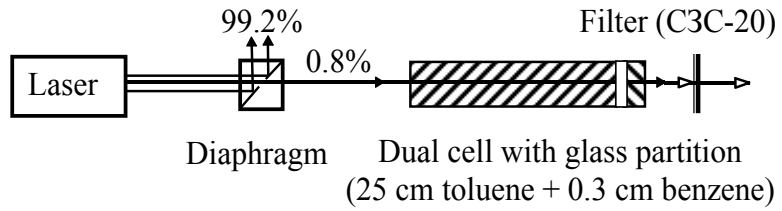

Fig. 4. Improved optical scheme for generation of the seed subnanosecond SRS pulse in benzene.

The coefficient of enhancement in the scheme (Fig. 3) equaled $k_{e n}=16$, which was a little lower than the expected value. However, after changing the toluene with benzene we obtained $k_{e n}=23$.

The dynamics of self-focusing can be more efficient in an improved optical scheme shown in Fig. 4. A dual cell filled with toluene and benzene, separated with a glass partition of $2 \mathrm{~mm}$ thickness, was used to generate the seed SRS pulse. The first section of the dual cell with toluene had the length $L_{1}=25 \mathrm{~cm}$, and the second one with benzene had the length $L_{2}=3 \mathrm{~mm}$.

At the output of the first section of the dual cell, the self-focusing area had a velocity close to a group speed $v_{g}$ of the laser radiation [10]. The group speed of light in toluene is $v_{g}=1.97 \cdot 10^{8} \mathrm{~m} / \mathrm{s}$. Under given conditions, the length of the focal region is approximately equal to $\ell_{f}=2 \mathrm{~cm}$ [9]. Consequently, the seed SRS pulse can be generated in the second section (filled with benzene, which has no expressed self-focusing properties) with the duration

$\tau_{S R S}=\left(\ell_{f}+L_{2}\right) / \mathrm{v}_{g}=0.12 \mathrm{~ns}$

Next, the seed pulse after the dual cell was amplified at a single pass through a benzene-filled cell with the length $1 \mathrm{~m}$ (Fig. 5). The coefficient of enhancement equaled $k_{e n}=20$.

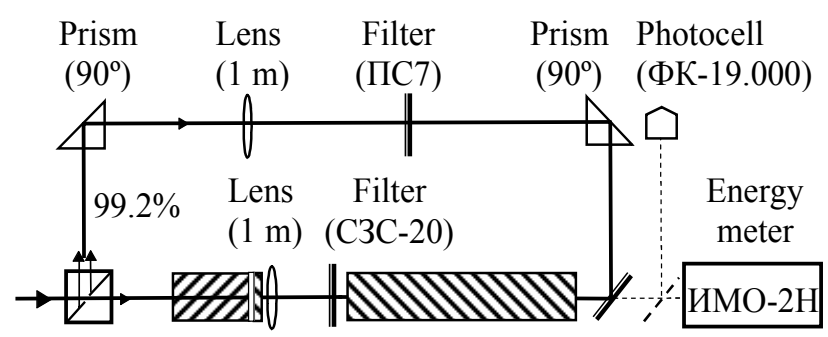

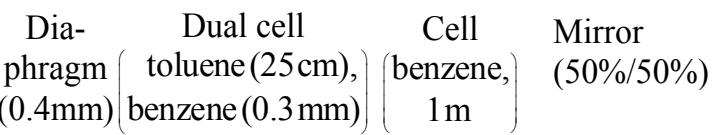

Fig. 5. Optical scheme for enhancement of SRS pulse from a single focal region of self-focusing in benzene. 


\section{Analysis and conclusion}

The experimental data (Fig. 2) allowed us to evaluate the effectiveness of SRS in a separate focal area. Assuming that the laser pulse has the duration $\tau_{L}=30 \mathrm{~ns}$ at the half level of intensity and the energy of $E_{L d}=4 \mathrm{~mJ}$ after the diaphragm, we obtain that the value of the laser power was $P_{L}=E_{L d} / \tau_{L}=133 \mathrm{~kW}$. The critical power of selffocusing in toluene is $P_{C R}=25 \mathrm{~kW}$ [9]. As the SRSthreshold and, hence, the threshold of self-focusing, in the absence of the diaphragm, has been exceeded by $\mu=3.5$ times, we can evaluate the power of the laser radiation in a separate focal area after entering the diaphragm: $P_{L}^{*}=k \mu P_{C R}=80 \mathrm{~kW}$, where $k=0.92$ is a loss factor due to the light reflection at the outer planes of the diaphragm. Thus, the power in the focal region equaled $80 \mathrm{~kW} / 133 \mathrm{~kW}=0.6$, i.e. $60 \%$ of the radiation that passed through the diaphragm. The other portion of the radiation $(40 \%)$ was not involved in the generation of SRS-pulse.

If the full depletion of the laser pulse at SRS takes place in the focal region [9], then the amplitude of the Stokes SRS pulse in the oscillograms must be also close to $60 \%$ of the laser pulse amplitude. The experimentally observed amplitude ratio of SRS/laser pulses (after taking into account the filter transmission and the spectral sensitivity of the photocell) reached $50 \%$. It can be concluded that the energy conversion efficiency of the laser radiation in the focal region reached the value of about $0.5 / 0.6 \approx 0.8(80 \%)$, while the quantum efficiency equaled $86 \%$.

Hence, achieving the high conversion efficiency of the laser radiation in SRS-active self-focusing fluids is principally possible. The effect of "absolute saturation of stimulated scattering" [6] has no fundamental nature.

The obtained value of the conversion efficiency indicates that it is incorrect to use approximations of a given excitation field and the field of the first Stokes SRS [11] or a given excitation field and the exponential growth of the first Stokes component [12] in order to describe the SRS at self-focusing. However, the model of deep depletion of the exciting radiation in the selffocusing focal areas [9] received the further experimental confirmation in our work.

The proposed and implemented scheme in Fig. 1 is suitable for generation of the seed sub-nanosecond ( $\sim 0.5 \mathrm{~ns}$ ) Stokes pulse. This pulse has been used for $\sim 20$ times compression of the giant laser pulses with a corresponding increase in power (Fig. 3).

The improved optical scheme with the dual cell (Fig. 4) makes it possible to generate the shorter ( $\sim .1 \mathrm{~ns})$ seed sub-nanosecond SRS pulse. This pulse has also been used for $\sim 20$ times compression (the optical scheme in Fig. 5).

Some limitation of the conversion efficiency in the amplification stage is mainly caused by the stimulated Mandelstam-Brillouin scattering, since a backward SRS is not significant [13].

\section{References}

1. J. Yao, Y. Wang, Nonlinear Optics and Solid-State Lasers: Advanced Concepts, Tuning-Fundamentals and Applications. Berlin-London-New York, Springer, 2012.

2. R.W. Boyd, S.G. Lukishova, Y.R. Shen, Selffocusing: Past and Present. Fundamentals and Prospects. New York, Springer, 2009.

3. S.V. Chekalin, V.P. Kandidov, From self-focusing light beams to femtosecond laser pulse filamentation // Uspekhi Fizicheskikh Nauk, 183(2), p. 133-152 (2013), in Russian.

4. E.J. Woodbury, W.K. Ng, Ruby laser operation near IR // Proc. IRE, 50, p. 2367-2369 (1962).

5. A.I. Ivanisik, V.I. Malyi, G.V. Ponezha, Spatialangular structure of anti-Stokes radiation formed by stimulated Raman scattering in a Kerr liquid // Optics and Spectroscopy, 80(2), p. 185-189 (1996).

6. A.I. Sokolovskaya, A.D. Kudryavtseva, G.L. Brekhovskikh, New optical phenomena at Raman light scattering in condensed matter // Proc. FIAN (Institute of Physics of the Academy of Sciences), 212, p. 16-37 (1991), in Russian.

7. V.I. Grygoruk, A.I. Ivanisik, P.A. Korotkov, Experimental Laser Optics. Kyiv, Publishingprinting centre "Kyiv University", 2007 (in Ukrainian).

8. A.I. Ivanisik, V.I. Malyi, G.V. Ponezha, Stimulated Raman scattering in self-focusing environments: New methods for experimental studies // Bulletin of Taras Shevchenko National University of Kyiv. Series: Physics \& Mathematics, 4, p. 239-248 (1997), in Ukrainian.

9. A.I. Ivanisik, V.I. Malyi, G.V. Ponezha, Effect of self-focusing on the angular spectra of stimulated Raman scattering // Optics and Spectroscopy, 85(1), p. 78-84 (1998).

10. A.I. Ivanisik, P.A. Korotkov, G.V. Ponezha, Temporal dynamics of focal point location under self-focusing of nanosecond laser pulses // Ukr. J. Phys. Opt. 15(1), p. 1-8 (2014).

11. V.N. Lugovoi, A.M. Prokhorov, Contribution to the theory of stimulated Raman scattering in focused light beams // Zhurnal eksperiment. teoretich. fiziki, 42(1), p. 42-46 (1975), in Russian.

12. G.V. Venkin, D.N. Klyshko, L.L. Kulyuk, Angular structure of higher components of stimulated Raman scattering of light // Kvantovaia elektronika, 7(5), p. 550-553 (1977), in Russian.

13. M.V. Dmitriev, O.Iu. Isaienko, A.I. Ivanisik, P.A. Korotkov, Experimental and theoretical study of stimulated Raman scattering indicatrix asymmetry // Semiconductor Physics, Quantum Electronics and Optoelectronics, 15(1), p. 44-47 (2012). 